\title{
Convergence and inversion theorems for generalized Weierstrass transform
}

\author{
A. K. Thakur ${ }^{1, a^{*}}$, Preeti Tamrakar ${ }^{2}$ \\ $1^{1 *}$ Department of Mathematics, Dr. C. V. Raman University, Bilaspur (C.G.) India-495001 \\ ${ }^{2}$ Research Scholar, Dr. C. V. Raman University, Bilaspur (C.G.) India-495001 \\ a thakur.amarnath968@gmail.com
}

Keywords: Laplace transforms, Stieltje'stransform, Whittaker's function, Recurrence relation.

Abstract. In this paper, we study the Weierstrass Transformation of certain generalized functions and their convolution transformation of certain generalised function and its inversion. We extend such type of results of convergence and inversion theorems for generalized weierstrass transforms (1.1) and (1.2).

\section{Introduction}

The Laplace transform, become of its simple exponential kernel and is dense with its generalizations. The Stieltje's transform, because of an outcome of iteration of Laplace transform is in now way poorer in respect to generalizations analogous to these, the Weierstrass transforms,

$$
f(s)=\int_{-\infty}^{\infty} K(s-t, 1) \varphi(t) d t
$$

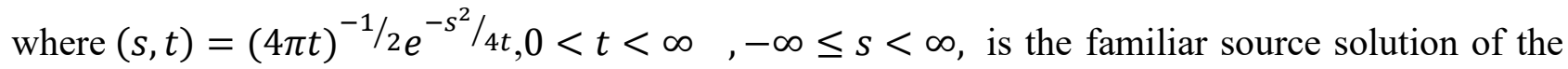
heat equation $\frac{\partial^{2} u}{\partial s^{2}}=\frac{\partial u}{\partial t}$, a particular case of convolution transform, $f(s)=\int_{a}^{b} K(s-$ $t) \varphi(t) d t, s$ is complex, because of it involving the exponential of the square of the difference of the variable of the transform and that of the integrand, may be thought for generalization with the help of a special function which Can be particularized to exponential function. We have considered the following generalization of Weierstrass transforms by using Whittaker's function

$$
\begin{aligned}
& f(x)=\sqrt{\frac{1}{2 \pi t^{1 / 2}}} \int_{-\infty}^{\infty} \frac{\frac{e^{-(x-y)^{2}}}{8 t}}{\sqrt{x-y}} W_{v / 2+\frac{1}{4}, \frac{1}{4}\left\{\frac{(x-y)^{2}}{4 t}\right\}} d \alpha(y) \\
& f(x)=2^{-3 / 4} \pi^{-1 / 2} \int_{-\infty}^{\infty}(x-y)^{\frac{-1}{2}} w_{\frac{v}{2}+\frac{1}{4}, \frac{1}{4}\left\{\frac{(x-y)^{2}}{2}\right\}} \varphi(y) d y
\end{aligned}
$$

\section{The convergence theorems on (1.1)}

\section{Theorem 1.}

If the integral (1.1) converges to a value $\mathrm{A}$ when $\mathrm{x}=\mathrm{x}_{0}, \mathrm{t}=1$; then it converges for $\infty<x<$ $-\infty, 0<t<1$ and

$$
\lim _{t \rightarrow 1^{-}} \int_{-\infty}^{\infty} \frac{e^{-\left(x_{0-y}\right)^{2}} / 8 t}{\sqrt{x_{0}-y}} w_{v / 2+1 / 2,1 / 4} \frac{(x-y)^{2}}{4 t} d \propto(y)=A
$$

\section{Proof:}

We first quote a known lemma due to Hirschman and Widder [1]. If $\propto(\mathrm{x})$ is of bounded variation in $\mathrm{a} \leq x<R$ for every $\mathrm{R}>0, \propto(\infty)$ exists, $\beta(x)$ is positive, continuous and non-increasing in a $\leq x<\infty$, then

$$
\int_{a}^{\infty} \beta(x) d \propto(x)
$$


converges set for fixed $x, x_{0}, t$.

$$
\begin{gathered}
\alpha^{*}(\mathrm{y})=\int_{0}^{y} e^{-\left(x_{0}-z\right)^{2} / 8}\left[\mathrm{x}_{0}-\mathrm{z}\right]^{-1 / 2} w_{v / 2+1 / 4,1 / 4} \frac{\left(x_{0}-z\right)^{2}}{4} \mathrm{~d} \propto(\mathrm{z}) \\
\beta(y)=e^{-(x-y)^{2} / 8 t} e^{\left(x_{0}-y\right)^{2} / 8}\left[\frac{x_{0}-y}{x-y} t^{1 / 2}\right]^{1 / 2} \frac{w_{v / 2}+1 / 4,1 / 4 \frac{(x-y)^{2}}{4 t}}{w_{v / 2}+1 / 4,1 / 4 \frac{\left(x_{0}-y\right)^{2}}{4}}
\end{gathered}
$$

Now (1.1) is convergent for, $x=x_{0}, t=1$

$\Rightarrow \int_{-\infty}^{\infty} \frac{e^{-\left(x_{0}-y\right)^{2} / 8}}{\sqrt{x_{0}}-y} w_{v / 2}+1 / 4,1 / 4 \frac{\left(x_{0}-y\right)^{2}}{4} \mathrm{~d} \propto(\mathrm{y})$ is convergent.

$\Rightarrow\left(\int_{-\infty}^{0}+\int_{0}^{\infty}\right) \frac{e^{-\left(x_{0}-y\right)^{2} / 8}}{\sqrt{x_{0}}-y} w_{v / 2}+1 / 4,1 / 4 \frac{\left(x_{0}-y\right)^{2}}{4} \quad \mathrm{~d} \propto(\mathrm{y})$ is convergent.

$\Rightarrow \int_{0}^{\infty} \frac{e^{-\left(x_{0}-y\right)^{2} / 8}}{\sqrt{x_{0}}-y} w_{v / 2}+1 / 4,1 / 4 \frac{\left(x_{0}-y\right)^{2}}{4} \mathrm{~d} \propto(\mathrm{y})$ is convergent.

$\Rightarrow \propto^{*}(\infty)$ exists.

Again since $\beta(y)$ is continuous and $\alpha^{*}(y)$ is of bounded variation is every finite interval, therefore

$$
\frac{1}{(2 \pi t)^{1 / 2}} \int_{0}^{y_{1}} \beta(y) d \propto^{*}(y) \text { is convergent , }\left(0<y_{1}<\infty\right) \text {. }
$$

Also $w_{v / 2}+1 / 4,1 / 4\left(z^{2} / 2\right) \quad \mathrm{o}\left(z^{2} e^{\frac{-z^{2}}{2}}\right) \quad$ for large, $|z|$.

$\Rightarrow w_{v / 2}+1 / 4,1 / 4\left(z^{2} / 2\right) \quad$ is positive, continuous and decreasing.

$\Rightarrow \beta(y)$ is positive, continuous and decreasing.

$\Rightarrow \int_{y_{1}}^{\infty} \beta(y) \mathrm{d} \alpha^{*}(\mathrm{y})$ is convergent by the lemma quoted.

$$
\Rightarrow \frac{1}{(2 \pi t)^{1 / 2}} \int_{y_{1}}^{\infty} \beta(y) d \alpha^{*}(y)
$$

is convergent. (2.5) and (2.6) $\Rightarrow \frac{1}{(2 \pi t)^{1 / 2}} \int_{0}^{\infty} \beta(y) d \propto^{*}(y)$ is convergent.

$$
\Rightarrow \frac{1}{\sqrt{ }\left(2 \pi t^{1 / 2}\right.} \int_{a}^{\infty} \frac{e^{-(x-y)^{2} / 8 t}}{\sqrt{x-y}} W_{\frac{v}{2}}+\frac{1}{4}, \frac{1}{4} \frac{(x-y)^{2}}{4 t} d \propto(y)
$$

is convergent. $-\infty<x<\infty, 0<t<1$ by the equations (2.3) and (2.4). Consider the integral

$$
\frac{1}{(2 \pi \sqrt{t})^{1 / 2}} \int_{-\infty}^{0} \frac{e^{-(x-y)^{2} / 8 t}}{\sqrt{x}-y} w_{v / 2}+1 / 4,1 / 4 \frac{(x-y)^{2}}{4 t} \mathrm{~d} \propto(\mathrm{y})
$$

Which on making the substitution $\frac{x-y}{\sqrt{2 t}}=\mathrm{z}$ equals to

$\frac{1}{(2 \pi \sqrt{ } t)^{1 / 2}} \int_{\infty}^{x / \sqrt{2} t} \frac{e^{-z^{2} / 4}}{\sqrt{z \sqrt{2 t}}} w_{v / 2}+1 / 4,1 / 4\left[z^{2} / 2\right] \mathrm{d} \alpha(\mathrm{x}-\mathrm{z} \sqrt{2} t)$

$=-\frac{1}{\sqrt{2 \pi \sqrt{t}}} \int_{x / \sqrt{2} t}^{\infty} \frac{e^{-z^{2} / 4}}{\sqrt{z \sqrt{2 t}}} w_{v / 2}+1 / 4,1 / 4\left[z^{2} / 2\right] \mathrm{d} \alpha(\mathrm{x}-\mathrm{z} \sqrt{2} t)$

which is convergent by the lemma and therefore (2.8) is convergent for

$-\infty<\mathrm{x}<\infty 0<t<1$. (2.7) and (2.8) $\Rightarrow$

(1.1) is convergent for $-\infty<\mathrm{x}<\infty, 0<t<1$.

To attend the second part of the theorem, we proceed as under

$=\frac{1}{\sqrt{2 \pi \sqrt{t}}}\left[\int_{-\infty}^{x_{0}} \frac{e^{-\left(x_{0}-y\right)^{2} / 8 t}}{\sqrt{x_{0}-y}} w_{v / 2}+1 / 4,1 / 4 \frac{\left(x_{0}-y\right)^{2}}{4 t} \mathrm{~d} \propto(y)\right.$

$$
\left.+\int_{x_{0}}^{\infty} \frac{e^{-\left(y-x_{0}\right)^{2} / 8 t}}{\sqrt{y-x_{0}}} w_{v / 2}+1 / 4,1 / 4 \frac{\left(y-x_{0}\right)^{2}}{4 t} \mathrm{~d} \alpha(y)\right]
$$

$=\frac{1}{\sqrt{2 \pi \sqrt{t}}}\left[\int_{\infty}^{0} e^{-z / 8 t} z^{-1 / 4} w_{v / 2}+1 / 4,1 / 4\left(\frac{z}{4 t}\right) d \propto\left(x_{0}-\sqrt{z}\right)\right.$

$\left.+\int_{0}^{\infty} e^{-z / 8 t} z^{-1 / 4} w_{v / 2}+1 / 4,1 / 4\left(\frac{z}{4 t}\right) d \propto\left(x_{0}+\sqrt{z}\right)\right]$

$=\frac{1}{\sqrt{2 \pi \sqrt{t}}} \int_{a}^{\infty} e^{-z / 8 t} z^{-1 / 4} w_{v / 2}+1 / 4,1 / 4\left(\frac{z}{4 t}\right)\left[d \propto\left(x_{0}+\sqrt{z}\right)-d \propto\left(x_{0}-\sqrt{z}\right)\right]$ 
Which is a generalized Laplace transforms converging for $t=1$ by the hypothesis. Since it represents a continuous function of $\mathrm{t}$ in $0<\mathrm{t}<1$, therefore its value at $\mathrm{t}=1$ will be equal to its limit for $\mathrm{t}=1^{-}$. This is Convergence theorem for (1.1).

\section{Corollary}

If we take $v=0$, then we get the convergence theorem Hirschman and Widder [1].

\section{The convergence problems on (1.2)}

\section{Theorem 2.}

If (1.2) be convergent for some $\mathrm{x}=x_{0}$ and

then,

$$
\int_{a}^{y}[\varphi(z)-\varphi(a)] d z=0,|y-a|, \mathrm{y} \rightarrow \mathrm{a}
$$

$$
\begin{aligned}
& \lim _{t \rightarrow 0^{+}} 2^{-3 / 4} \pi^{-1 / 2} t^{-1 / 4} \int_{-\infty}^{\infty}(a-y)^{-1 / 2} w_{v / 2}+1 / 4,1 / 4 \frac{(a-y)^{2}}{2 t} \varphi(y) d y \\
= & \frac{\Gamma\left(\frac{1}{2}\right)}{\Gamma\left(\frac{1}{2}-\frac{v}{2}\right)} \varphi(a)
\end{aligned}
$$

$\varphi(x)$ being absolutely integrable in every finite integral and $v$ being a non - positive real no.

\section{Proof}

We first prove the following lemma. If (1.2) be convergent for some $x=x_{0}$ and

$$
\begin{aligned}
& \int_{a}^{y}[\varphi(z)-\varphi(a)] d z=a(y-a), \mathrm{y} \rightarrow a^{+} \\
& \quad \lim _{t \rightarrow 0^{+}} 2^{-3 / 4} \pi^{-1 / 2} t^{-1 / 4} \int_{a}^{\infty}(a-y)^{-1 / 2} w_{v / 2}+1 / 4,1 / 4 \frac{(a-y)^{2}}{2 t} \varphi(y) d y
\end{aligned}
$$

$\lim _{t \rightarrow 0^{+}} 2^{-3 / 4} \pi^{-1 / 2} t^{-1 / 4} \int_{-\infty}^{a}(a-y)^{-1 / 2} w_{v / 2}+1 / 4,1 / 4 \frac{(a-y)^{2}}{2 t} \varphi(y) d y$

$=\frac{\Gamma(1 / 2)}{\Gamma(1 / 2-v / 2)} \cdot \frac{\varphi(a)}{2}$.

For this we choose $\delta>0$ and write (3.4) as under

$t^{-1 / 4} 2^{-3 / 4} \pi^{-1 / 2}\left[\int_{a}^{a+\delta}+\int_{a+\delta^{*}}^{\infty}\right](a-y)^{-1 / 2} w_{v / 2}+1 / 4,1 / 4 \frac{(a-y)^{2}}{2 t} \varphi(y) d y=I_{1}(t)+I_{2}(t)$.

Now set

(3.6), in the light of Slater [2]

$$
\begin{aligned}
& \propto(\mathrm{y})=\int_{a+\delta}^{y}\left(x_{0}-z\right)^{-1 / 2} w_{v / 2}+1 / 4,1 / 4 \frac{\left(x_{0}-z\right)^{2}}{2} \varphi(z) d z \\
& \text { and } \beta(y)=\frac{\left(\frac{a-y}{\sqrt{t}}\right)^{-1 / 2} w_{v / 2}+1 / 4,1 / 4 \frac{(a-y)^{2}}{2 t}}{\left(x_{0}-y\right)^{-1 / 2} w_{v / 2}+1 / 4,1 / 4 \frac{\left(x_{0}-y\right)^{2}}{2}}
\end{aligned}
$$

$w_{k, m^{(x)}} \sim x^{k} e^{-x / 2} \Rightarrow$

$\beta(y)=\frac{1}{t^{v / 2}}\left(\frac{a-y}{x_{0}-y}\right)^{v} \frac{e^{\left(x_{0}-y\right)^{2 / 4}}}{\left.e^{(a-y)}\right)^{2 / 4 t}}$

$\Rightarrow \beta(y)$ is decreasing and $\beta(y) \rightarrow 0$, as $\mathrm{y} \rightarrow \infty$,

since the denominator is more rapidly growing function as $\mathrm{t} \rightarrow 0^{+}$.

We have, $I_{2}(\mathrm{t})=2^{-3 / 4}(\pi \mathrm{t})^{-1 / 2} \int_{a+\delta}^{\infty} \beta(y) \mathrm{d} \propto(\mathrm{y})$ by the equations (3.5) and (3.6)

$$
\begin{aligned}
& =2^{-3 / 4}(\pi \mathrm{t})^{-1 / 2}\left[\{\beta(\mathrm{y}) \propto(\mathrm{y})\}_{a+\delta}^{\infty}-\int_{a+\delta}^{\infty} \propto(y) \mathrm{d} \beta(y)\right] \\
& =-2^{-3 / 4}(\pi \mathrm{t})^{-1 / 2} \int_{a+\delta}^{\infty} \alpha(y) \mathrm{d} \beta(\mathrm{y}), \text { since } \propto(\mathrm{a}+\delta)=0 \text { and } \beta(y) \rightarrow 0 \text { as } \mathrm{y} \rightarrow \infty
\end{aligned}
$$


$\Rightarrow\left|I_{2}(t)\right| \leq 2^{-3 / 4}(\pi t)^{-1 / 2} \mathrm{M} \int_{a+\delta}^{\infty}|\mathrm{d}\{-\beta(\mathrm{y})\}| .|\alpha(\mathrm{y})| \leq \mathrm{M}$

$=\sigma(1) \quad$ as $\mathrm{t} \rightarrow 0^{+} \quad$ by (3.6).

Again

$I_{2}(\mathrm{t})=2^{-3 / 4}(\pi \mathrm{t})^{-1 / 2} \int_{a}^{a+\delta}\left(\frac{a-y}{\sqrt{t}}\right)^{-1 / 2} w_{v / 2}+1 / 4,1 / 4 \frac{(a-y)^{2}}{2 t} \varphi(y) \mathrm{dy}$

$=2^{-3 / 4}(\pi \mathrm{t})^{-1 / 2} \int_{a}^{a+\delta}\left(\frac{a-y}{\sqrt{t}}\right)^{-1 / 2} w_{v / 2}+1 / 4,1 / 4 \frac{(a-y)^{2}}{2 t} e^{(a-y)^{2 / 4 t}} e^{-(a-y)^{2 / 4 \mathrm{t}}} \varphi(y) \mathrm{dy}$

$=2^{-3 / 4}(\pi t)^{-1 / 2} 2^{-v / 2-1 / 4} \int_{a}^{a+\delta} D_{v}\left(\frac{a-y}{\sqrt{t}}\right) e^{(a-y)^{2} / 4 t} e^{-(a-y)^{2 / 4 t}} \varphi(y) \mathrm{dy}$

$=2^{-v / 2-1}(\pi t)^{-1 / 2} \int_{a}^{a+\delta} D_{v}\left(\frac{a-y}{\sqrt{t}}\right) e^{(a-y)^{2} / 4 t} e^{-(a-y)^{2} / 4 \mathrm{t}} \varphi(y) \mathrm{dy}$.

Now, $\frac{d}{d x}\left[D_{v}(x) e^{x^{2 / 4}}\right]=e^{x^{2 / 4}}\left[D_{v}(x)+v / 2 D_{v}(x)\right]$

$=e^{x^{2 / 4}} \cdot v D_{v-1}(\mathrm{x})$ by using the recurrence relation $D_{v}(x)+v / 2 D_{v}(x)-v D_{v-1}(\mathrm{x})=0$

$=\mathrm{a}-$ ve quantity in our case.

Therefore $D_{v}(x) e^{x^{2 / 4}}$ is decreasing and hence applying mean value Theorem Widder [3] to $I_{1}(\mathrm{t})$, we get

$I_{1}(\mathrm{t})=2^{-v / 2-1}(\pi t)^{-1 / 2} D_{v}(0) \int_{a}^{\varepsilon} \frac{e^{-(y-a)^{2}}}{4 t} \varphi(y) \mathrm{dy},(\mathrm{a} \leq \varepsilon \leq a+\delta)$

$=2^{-v / 2-1} \frac{(1 / 2) 2^{v / 2}}{(1 / 2-v / 2)} \int_{a}^{\varepsilon} \frac{e^{-(y-a)^{2}}}{4 t} \varphi(y) \mathrm{dy}$.

To estimate $\int_{a}^{\varepsilon} e^{-(y-a)^{2}} / 4 t \varphi(y)$ dy, we use Widder [4] with

$\mathrm{K}=\frac{1}{t}, h(y)=-(y-a)^{2} / 4$, and get $(4 \pi t)^{-1 / 2} \int_{a}^{\varepsilon} e^{-(y-a)^{2}} / 4 t \varphi(y) \mathrm{dy}, \sim \frac{\varphi(a)}{2}\left(\mathrm{t} \rightarrow 0^{+}\right)$.

Thus $I_{1}(\mathrm{t})=\frac{\Gamma(1 / 2)}{\Gamma(1 / 2-v / 2)} \cdot \frac{\varphi(a)}{2},\left(\mathrm{t} \rightarrow 0^{+}\right)$

$$
I_{1}(t)+I_{v}(t)=\frac{\Gamma(1 / 2)}{\Gamma(1 / 2-v / 2)} \cdot \frac{\varphi(a)}{2} .
$$

Similarly by putting $-y$ for $y$ and setting

$$
\begin{aligned}
& \alpha^{*}(\mathrm{y})=\int_{-(a+\delta)}^{y}\left(x_{0}-z\right)^{-1 / 2} \mathrm{~W} v_{/ 2}+1 / 4,1 / 4 \frac{1}{2}\left(x_{0}-z\right)^{2} \varphi(z) d z \\
& \beta^{*}(\mathrm{y})=\mathrm{t}^{-1 / 4}(\mathrm{a}+\mathrm{y})^{-1 / 2} \mathrm{~W} v_{/ 2}+1 / 4,1 / 4 \frac{(a+y)^{2}}{2 t} /\left(\mathrm{x}_{0}-\mathrm{y}\right)^{-1 / 2} \mathrm{~W} v_{/ 2}+1 / 4,1 / 4 \frac{1}{2}\left(x_{0}-z\right)^{2}
\end{aligned}
$$

and proceeding as usual, we get

$$
\begin{aligned}
& \lim _{t \rightarrow 0^{+}} 2^{-3 / 4} \pi^{-1 / 2} t^{-1 / 4} \int_{-\infty}^{a}(a-y)^{-12} \mathrm{~W} v_{/ 2}+1 / 4,1 / 4 \frac{(a-y)^{2}}{2 t} \cdot \varphi(\mathrm{y}) \mathrm{dy} \\
& \frac{\Gamma(1 / 2)}{\Gamma(1 / 2-v / 2)} \cdot \frac{\varphi(a)}{2} \quad \text { (3.7) and (3.8) }=>\text { (3.2).Hence, the theorem. }
\end{aligned}
$$

\section{Corollary:}

If we take $v=0$, then we get the theorem established by Hirschman and Widder [4].

\section{The inversion based problems on (1.1)}

\section{Theorem 4.}

If $\varphi(x) \varepsilon c$ at $\mathrm{x}=\mathrm{a}$ and if

$$
\left.\mathrm{u}(\mathrm{x}, \mathrm{t})=\frac{1}{\sqrt{2 \pi \sqrt{t}}} \int_{-\infty}^{\infty} e^{-(\mathrm{x}-\mathrm{y})^{2} / 8 \mathrm{t}} / \sqrt{(x}-y\right) \mathrm{W} v_{/ 2}+1 / 4,1 / 4 \frac{(x-y)^{2}}{8 t} \varphi(\mathrm{y}) \mathrm{dy}
$$

converges at $\left(x_{0}, 1\right)$, then

$$
\lim _{t \rightarrow 0^{+}} \mathrm{u}(\mathrm{x}, \mathrm{t})=\frac{\varphi(a)}{\Gamma\left(1-\frac{v}{2}\right)} \quad(v \leq 0) .
$$




\section{Proof}

Choose an arbitrary $\delta>0$ and decompose (4.1) into three integrals such as $\mathrm{I}_{1}(\mathrm{x}, \mathrm{t}), \mathrm{I}_{2}(\mathrm{x}, \mathrm{t})$ and $\mathrm{I}_{3}(\mathrm{x}$, t) corresponding to the intervals $(-\infty, \mathrm{a}-\delta),(\mathrm{a}-\delta, \mathrm{a}+\delta)$,

$(\mathrm{a}+\delta, \infty)$ respectively.

\section{Case I:}

Let $x_{0} \leq \mathrm{a}+\delta$ and set

$$
\propto(\mathrm{y})=\int_{a+\delta}^{y} e^{-(\mathrm{x} 0-\mathrm{z})^{2} / 8}\left(\mathrm{x}_{0}-\mathrm{z}\right)^{-1 / 2} \mathrm{~W}_{\mathrm{V} / 2}+1 / 4,1 / 4 \frac{1}{4}\left(\mathrm{x}_{0}-\mathrm{y}\right)^{2} \varphi(\mathrm{z}) \mathrm{dz}
$$

$\beta(\mathrm{y})=\mathrm{e}^{-(\mathrm{x}-\mathrm{y})^{2} / 8 \mathrm{t}} \mathrm{e}^{(\mathrm{x} 0-\mathrm{y})^{2} / 8}\left[\frac{x-y}{x-y} \sqrt{t}\right]^{-1 / 2}$

$$
=\mathrm{W}_{\mathrm{V} / 2}+1 / 4,1 / 4 \frac{1}{4 t}\left(\mathrm{x}_{0}-\mathrm{y}\right)^{2} / \mathrm{W} v_{/ 2}+1 / 4,1 / 4 \frac{1}{4}\left(\mathrm{x}_{0}-\mathrm{y}\right)^{2} \text {. }
$$

Then, $\mathrm{I}_{3}(\mathrm{x}, \mathrm{t})=\frac{1}{\sqrt{2 \pi}} \int_{a+\delta}^{\infty} \beta(y) \mathrm{d} \alpha(\mathrm{y})$

$=\frac{1}{\sqrt{2 \pi}} \int_{a+\delta}^{\infty} \alpha(y) \mathrm{d}[-\beta(\mathrm{y})]$ by integration by parts, since $\alpha(\mathrm{a}+\delta)=0$ and $\beta(y) \rightarrow 0$

as $\mathrm{y} \rightarrow \infty$. This

$\Rightarrow\left|I_{3}(x, t)\right| \leq \frac{\mathrm{M}}{\sqrt{2 \pi}}|\beta(a+\delta)| \quad(|\propto(y)| \leq M)$

$\Longrightarrow I_{3}(x, t) \rightarrow 0$ as $\mathrm{x} \rightarrow \mathrm{a}$ and $\mathrm{t} \rightarrow 0^{+}$by (4.4).

\section{Case II:}

Let $\mathrm{x}_{0}>\mathrm{a}+\delta$ and say $\mathrm{x}_{0}=\mathrm{a}+\delta_{1}$ then

$I_{3}(x, t)=\int_{a+\delta}^{\infty}=\int_{a+\delta}^{a+\delta_{1}}+\int_{a+\delta_{1}}^{\infty}=I_{3}(x, t)+I_{3}(x, t)$ (say).

Now $\lim _{\mathrm{t} \rightarrow 0^{+}} I_{3}(x, t)=0$ as in case-I.

$\operatorname{ForI}_{3}(x, t)$, we set

$$
\begin{aligned}
\propto(\mathrm{y}) & =\int_{a+\delta}^{y} e^{-(\mathrm{z}-\mathrm{x} 0)^{2} / 8}\left(\mathrm{z}-\mathrm{x}_{0}\right)^{-1 / 2} \mathrm{~W} v_{/ 2}+1 / 4,1 / 4 \frac{1}{4}(\mathrm{z}-)^{2} \varphi(\mathrm{z}) \mathrm{dz} . \\
\beta(\mathrm{y}) & =\mathrm{e}^{-(\mathrm{y}-\mathrm{x})^{2} / 8 \mathrm{t}} e^{(\mathrm{z}-\mathrm{x} 0)^{2} / 8}\left[\frac{y-x_{0}}{y-x} t^{v / 2}\right]^{-1 / 2}=\frac{\mathrm{W} v / 2+1 / 4,1 / 4 \frac{1}{4 t}(\mathrm{y}-\mathrm{x})^{2}}{\mathrm{~W} v / 2+1 / 4,1 / 4 \frac{1}{4}\left(\mathrm{y}-\mathrm{x}_{0}\right)^{2}} .
\end{aligned}
$$

Then $I_{3}(x, t)=\frac{1}{\sqrt{2 \pi}} \int_{a+\delta}^{a+\delta_{1}} \beta(y) d \alpha(y)$

This $\Rightarrow\left|I_{3}(x, t)\right| \leq \frac{M}{\sqrt{2 \pi}}|\beta(a+\delta)| \quad(|\alpha(y)| \leq M)$

$\Rightarrow I_{3}(x, t) \rightarrow 0$, as $\mathrm{x} \rightarrow \mathrm{a}$ and $\mathrm{t} \rightarrow 0^{+}$by (4.6).

Now, $\lim _{t \rightarrow 0^{+}}^{x \rightarrow a}\left|I_{3}(x, t)-I_{1}(x, t)\right|=$

$\lim _{t \rightarrow 0^{+}}^{x \rightarrow a}\left|\frac{1}{\sqrt{2 \pi \sqrt{ } t}}\left[\left[\int_{a+\delta}^{\infty}-\int_{-\infty}^{a-b}\right]\right] \mathrm{e}^{-(\mathrm{x}-\mathrm{y})^{2} / 8 \mathrm{t}} / \sqrt{\mathrm{x}}-\mathrm{yW} v_{/ 2}+1 / 4,1 / 4 \frac{1}{4 t}(\mathrm{x}-\mathrm{y})^{2} \varphi(\mathrm{y}) \mathrm{dy}\right|$

$\lim _{t \rightarrow 0^{+}}^{x \rightarrow a} \frac{N}{\sqrt{2 \pi \sqrt{t}}} \int_{\text {Iy-a I } \geq \delta} \mathrm{e}^{-(\mathrm{x}-\mathrm{y})^{2} / 8 \mathrm{t} / \sqrt{\mathrm{x}}-\mathrm{y}}\left|\mathrm{W} v_{/ 2}+1 / 4,1 / 4 \frac{1}{4 t}(\mathrm{x}-\mathrm{y})^{2} \quad\right| \mathrm{dy},|\varphi(y)| \leq N$

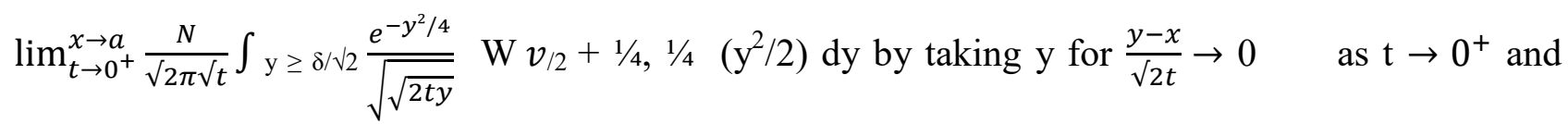
$\mathrm{y} \rightarrow \infty$ by using the asymptotic behaviour of $\mathrm{W}_{\mathrm{k}, \mathrm{m}}(\mathrm{z})$.

$\lim _{t \rightarrow 0^{+}}^{x \rightarrow a} I_{3}(x, t)=\lim _{t \rightarrow 0^{+}}^{x \rightarrow a} I_{1}(x, t)$.

Again $\left|\mathrm{I}_{2}(\mathrm{x}-\mathrm{t})-\frac{\varphi(a)}{\Gamma\left(1-\frac{v}{2}\right)}\right|=\mid \frac{1}{\sqrt{2 \pi \sqrt{t}}} \int_{a-\delta}^{a+\delta} e^{-(\mathrm{x}-\mathrm{y})^{2} / 8 \mathrm{t}} / \sqrt{x}-y \mathrm{~W} v_{/ 2}+1 / 4,1 / 4 \frac{1}{4 t}(\mathrm{x}-\mathrm{y})^{2}[\varphi(y)-\varphi(a)+$ $\varphi(a)] \mathrm{dy}-\frac{\varphi(a)}{(1-v / 2)} \mid$

$\leq 1 . u \cdot b_{|y-a| \leq \delta}|\varphi(y)-\varphi(a)| \frac{1}{\sqrt{2} \pi \sqrt{t}} \int_{a-\delta}^{a+\delta} \mathrm{e}^{-(\mathrm{x}-\mathrm{y})^{2} / 8 \mathrm{t}} / \sqrt{\mathrm{x}}-\mathrm{y} \mathrm{W} v_{/ 2}+1 / 4, \quad 1 / 4 \frac{1}{4 t}(\mathrm{x}-\mathrm{y})^{2} \mathrm{dy} \quad+\varphi(a) \quad \mid$ $\frac{1}{\sqrt{2 \pi \sqrt{t}}} \int_{a-\delta}^{a+\delta} \frac{e^{-(\mathrm{x}-\mathrm{y})^{2} / 8 \mathrm{tt}}}{\sqrt{x}-y} \mathrm{~W} v_{/ 2}+1 / 4,1 / 4 \frac{1}{4 t}(\mathrm{x}-\mathrm{y})^{2} \mathrm{dy}-\frac{1}{\Gamma\left(1-\frac{v}{2}\right)} \mid$

$\leq 1 . u . b_{|y-a| \leq \delta}|\varphi(y)-\varphi(a)|\left|2^{-v / 2-1} \pi^{-1 / 2} \int_{\frac{x(a+\delta)}{\sqrt{2} t}}^{\frac{x(a-\delta)}{\sqrt{2} t}} e^{-\mathrm{y}^{2} / 4} \mathrm{D} v_{(\mathrm{y})} \mathrm{dy}\right|$ 


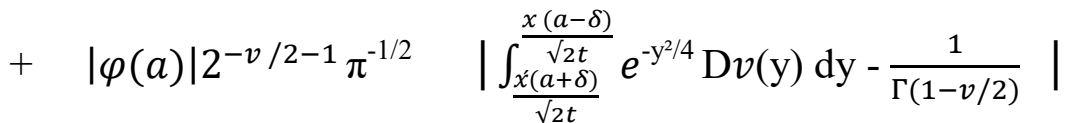

Now, since $\frac{x-(a+\delta)}{\sqrt{2} t}<0$ and $\frac{x-(a-\delta)}{\sqrt{2} t}>0$

in $|x-a|<\delta / 2$, therefore

$\lim _{t \rightarrow 0^{+}}^{x \rightarrow a}\left|\mathrm{I}_{2}(\mathrm{x}-\mathrm{t})-\frac{\varphi(a)}{\Gamma\left(1-\frac{v}{2}\right)}\right| \leq \lim _{|y-a| \leq \delta} \frac{\varphi(y)-\varphi(a)}{\Gamma(1-v / 2)}$,

by using $\int_{-\infty}^{\infty} e^{-\mathrm{y}^{2} / 4} \mathrm{D} v(\mathrm{y}) \mathrm{dy}=\frac{\pi^{1 / 22^{(v+1) / 2}}}{\Gamma(1-v / 2)}$.

But $\lim _{|y-a| \leq \delta}|\varphi(y)-\varphi(a)|=0$ by continuity of $\varphi(x)$ at $\mathrm{x}=\mathrm{a}$.

Therefore $\lim _{t \rightarrow 0^{+}}^{x \rightarrow a}\left|\mathrm{I}_{2}(\mathrm{x}-\mathrm{t})-\frac{\varphi(a)}{\Gamma\left(1-\frac{v}{2}\right)}\right| \leq 0$,

i. e, $\lim _{t \rightarrow 0^{+}}^{x \rightarrow a} \mathrm{I}_{2}(\mathrm{x}-\mathrm{t})=\frac{\varphi(a)}{\Gamma\left(1-\frac{v}{2}\right)}$.

Thus $\mathrm{u}(\mathrm{x}, \mathrm{t})=\mathrm{I}_{1}(\mathrm{x}, \mathrm{t})+\mathrm{I}_{2}(\mathrm{x}, \mathrm{t})+\mathrm{I}_{3}(\mathrm{x}, \mathrm{t})$

$\Rightarrow \lim _{x \rightarrow 0}^{t \rightarrow 0} \quad(\mathrm{x}, \mathrm{t})=\lim _{t \rightarrow 0^{+}}^{x \rightarrow a} \mathrm{I}_{2}(\mathrm{x}-\mathrm{t})=\frac{\varphi(a)}{\Gamma\left(1-\frac{v}{2}\right)}$.

Hence, the theorem.

\section{Corollary:}

If we take $v=0$, we get the theorem established by Hirschman and Widder [5]

\section{Theorem 5.}

If $\varphi(y)$ be bounded and continuous in $-\infty<y<\infty$ and if

$$
\mathrm{f}(\mathrm{x})=\frac{1}{\sqrt{2 \pi}} \int_{-\infty}^{\infty} \frac{e^{-(x-\gamma)^{2} / 8}}{\sqrt{x}-y} \mathrm{~W} v_{/ 2}+1 / 4,1 / 4 \frac{1}{4}(\mathrm{x}-\mathrm{y})^{2} \varphi(y) \mathrm{dy} .
$$

Then $(-1)^{v} D^{-v} e-D^{2} f(x)=\varphi(x)$, where $v$ is an integer including zero.

\section{Proof}

According to pollard [6], theinversions of the integral transform

$\mathrm{f}(\mathrm{x})=\int_{-\infty}^{\infty} k(x-y) \varphi(y) d y$ is given by

$\mathrm{E}(\mathrm{D}) \mathrm{f}(\mathrm{x})=\varphi(x),\left(\mathrm{D}=\frac{d}{d x}\right)$, where $\frac{1}{E(s)}=h\left(\frac{s}{i}\right)$,

$\mathrm{h}(\mathrm{s})=\int_{-\infty}^{\infty} e^{-\mathrm{isx}} \mathrm{k}(\mathrm{x}) \mathrm{dx}$.

Therefore, $\mathrm{h}(\mathrm{s})$ for the transform (5.1) is given by

$\mathrm{h}(\mathrm{s})=\frac{1}{\sqrt{2 \pi}} \int_{-\infty}^{\infty} e^{-\mathrm{isx}} \frac{e^{-x^{2} / 8}}{\sqrt{x}} \mathrm{~W} v_{/ 2}+1 / 4,1 / 4\left(\frac{x^{2}}{4}\right) \mathrm{dx}$

$=(-1)^{v} / 2 \sqrt{\pi} \int_{-\infty}^{\infty} e^{-\mathrm{isx}} D^{v}\left(\mathrm{e}^{-\mathrm{x}^{2} / 4}\right) d x$ by using the differential representation of $\mathrm{W}_{\mathrm{k}, \mathrm{m}}$.

$=(-1)^{v} / 2 \sqrt{\pi} \int_{-\infty}^{\infty} e^{-\mathrm{isx}}\left(\mathrm{e}^{-\mathrm{x}^{2} / 4}\right) d x$ by integration by parts

$=(-1)^{v}(i s)^{v} \mathrm{e}^{-\mathrm{s}^{2}}$ by using the result Erdelyi [6, p.121].

Thus, the inversion function $\mathrm{E}(\mathrm{s})$ for the transform (5.1) is given by

$\int_{-\infty}^{\infty} e^{i x y}(i x)^{v} e^{-\alpha^{2} x^{2}} d x=\pi^{1 / 2} 2^{-v / 2} \alpha^{-(v+1)} e^{\frac{-y^{2}}{8 \alpha^{2}}} D_{v}\left(\frac{y}{\sqrt{2 \alpha}}\right)$,

$\operatorname{Re} \propto>0, v>-1, \arg (i x)=\frac{\pi}{2}(x>0)=-\frac{\pi}{2}(x>0)$

Therefore, the inversion function $\mathrm{E}(\mathrm{s})$ for the transform (5.1) is given by

$$
E(s)=(-1)^{v} s^{-v} e^{-s^{2}} \text {. }
$$

Therefore, the inversion operator $\mathrm{E}(\mathrm{D})$ is given by

$$
E(D)=(-1)^{v} D^{-v} e^{-D^{2}}
$$

Therefore, the inversion of (5.1) in the light of pollards result is given by

$$
(-1)^{v} D^{-v} e^{-D^{2}} f(x)=\varphi(x) \text {. }
$$




\section{Corollary:}

If we take $v=0$, we get the inversion theorem Hirschman.

\section{Remarks}

The Convergence and inversion theorems for generalized Weierstrass transforms are referred to as natural and conditional functions, respectively. Moreover, in their easily derivable special corollary, When $v=0$, would simplify considerably.

\section{References}

[1] I.I. Hirschman, and D.V. Widder, The convolution transform, Princeton Univ. Press, (1955), pp. 174-192.

[2] A. H. Zemanian, The convolution transformation of certain generalized $\mathrm{f}$ unctions and its inversion, Bull. Amer. Math. Soc. 72 (1966), 725-727.

[3] D. V. Widder, The Laplace transform, Princeton Univ.Press (1946), pp. 276.

[4] H. Pollard, An inv. formula for the St. tr. ,Duke. Math, Jl., vol. II, (1944), pp. 301-318.

[5] H. Pollard, The inv. of the tr. With reiterated St. Kernels, Duke Math. J1., vol. 14, (1947), pp. 129-142.

[6] A. Erdelyi , T.I.T. vol.I, McGraw Hill book Co. (1954)

[7] A. Erdelyi , T.I.T. vol.II, McGraw Hill book Co. (1954)

[6] V.I. Fabricant, Elementary evaluation of certain infinite integral involving Bessel function Quarterly of applied mathematics vol. LIX, no. 1, Canada, (2001), pp.1-24,

[7] A.K, Sinha, Applied Science periodical, Siwan, vol. XII, No-3 and vol. XII, no. 1 (2010).

[8] V.P.Mainara, A new generalization of the Laplace transforms Bull. of the cal. Math. Soc., vol. 53, pp. 23-31.

[9] H.M. Srivastava, A note on a generating function for the generalized Hermite polynomials, Nederl. Akad. Wetensch. Proc. Ser. A 79; Indag. Math. 38 (1976), pp. 457-461. 